\title{
LATTICES OVER ORDERS: FINITELY PRESENTED FUNCTORS AND PREPROJECTIVE PARTITIONS
}

\author{
BY
}

\author{
M. AUSLANDER AND S. O. SMALO
}

\begin{abstract}
Suppose $R$ is a commutative noetherian equidimensional Gorenstein ring and $\Lambda$ an $R$-algebra which is finitely generated as an $R$-module. A $\Lambda$-module $M$ is a lattice if $M_{p}$ is $\Lambda_{p}$-projective and $\operatorname{Hom}_{R}(M, R)_{p}$ is $\Lambda_{p}^{\mathrm{op}}$-projective for all nonmaximal prime $\overline{\overline{1}}$ deals $\bar{p}$ in $R$. We assume that $\Lambda$ is an $R$-order in the sense that $\Lambda$ is a lattice when viewed as a $\Lambda$-module. The first main result is to show that simple contravariant functors from lattices to abelian groups are finitely presented. This is then applied to showing that if $R$ is also local and complete, then the category of lattices has a preprojective partition. This generalizes previous results of the authors in the cases $R$ is artinian or a discrete valuation ring.
\end{abstract}

Introduction. In [6] it was proved that $\bmod \Lambda$, the category of finitely generated modules over an artin algebra $\Lambda$, has a preprojective partition, i.e., there is a unique collection of full subcategories $\left\{\underline{\underline{P}}_{i}\right\}_{i \in N \cup \infty}$ of ind $\Lambda$, the indecomposable $\Lambda$-modules, where $N$ is the nonnegative integers, having the following properties.

(a) If $A \in \underline{P}_{i}$ and $B \simeq A$, then $B \in \underline{P}_{i}$, for all $i$.

(b) $\underline{\underline{P}}_{i} \cap \underline{\underline{P}}_{j}=\varnothing$ for $i \neq j$ and $\cup_{i \in N \cup \infty} \underline{\underline{P}}_{i}=$ ind $\Lambda$.

(c) $\underline{\underline{P}}_{i}$ has only a finite number of nonisomorphic objects for each $i$ in $N$.

(d) For each $i$ in $N$, an indecomposable module $A$ is in $\underline{P}_{i}$ if and only if every surjective morphism $f: A \rightarrow B$ is a splittable surjection whenever every indecomposable summand of $B$ is in $\cup_{k \leqslant i} \underline{P}_{k}$.

What it means for a full subcategory of $\bmod \Lambda$ to have a preprojective partition was also similarly defined, and sufficient conditions for preprojective partitions to exist for a subcategory were given.

A general theory for lattices over orders was started in [1, 2], containing the classical definitions as a special case. (See $\$ 1$ for definition.) A category of lattices is a subcategory of a module category, and a preprojective partition can be defined analogously in this setting (and a preinjective partition by duality). The main purpose of this paper is to give the existence of such partitions for lattices over a rather general class of orders.

As for the case of artin algebras, an essential tool is the existence of left and right almost split morphisms. If $C$ is an indecomposable finitely generated nonprojective $\Lambda$-module for an artin algebra $\Lambda$, there exists an almost split sequence $0 \rightarrow A \rightarrow^{f} B$ $\rightarrow^{g} C \rightarrow 0$ [3], hence a right almost split morphism $g: B \rightarrow C$. That is, $g: B \rightarrow C$ is not a splittable epimorphism, and given $t: X \rightarrow C$ where $C$ is indecomposable and $t$ is

Received by the editors February 20, 1981.

1980 Mathematics Subject Classification. Primary 16A64; Secondary 13C10.

(C)1982 American Mathematical Society 0002-9947/82/0000-0609/\$04.25 
not an isomorphism, there is some $s: X \rightarrow B$ such that $g s=t$. If $P$ is indecomposable projective, there is trivially a right almost split morphism $i: r P \rightarrow P$, where $r$ denotes the radical of $\Lambda$. For lattices for orders over a complete local Gorenstein ring, the existence of almost split sequences was proved in [1, 2], hence giving the existence of right almost split morphisms for the indecomposable nonprojective lattices. Contrary to the situation for artin algebras, the case of indecomposable projective lattices is not trivial. It was known only when the dimension of the ground ring is at most 2 [2], so to imitate the procedure for artin algebras, we first have to prove the existence of right almost split morphisms $g: B \rightarrow C$, when $C$ is an indecomposable projective lattice for orders over a complete local Gorenstein ring.

Actually, in $\S 1$, we prove a more general theorem about simple functors being finitely presented, for lattices over arbitrary orders. In $\$ 2$ we give the existence of preprojective partitions. $\S 3$ is devoted to some generalizations of the results of $\S 1$.

We finally point out that in the case of classical orders, also the main theorems on preprojective partitions proved in [6] have analogous formulations, and this is proved in [7].

1. Simple functors are finitely presented. In this section we shall prove that simple functors not vanishing on projectives, from the category of lattices over orders in the general sense introduced in $[1,2]$ to abelian groups, are finitely presented.

We start out by recalling the general definition of lattices over orders, which contains the classical case of lattices over orders and finitely generated modules over artin algebras as special cases [2]. First we recall, for the convenience of the reader, some of the concepts needed to state the definition.

Let $R$ be a commutative noetherian ring. $R$ is equidimensional if $\operatorname{dim} R_{m}=\operatorname{dim} R$ for all maximal ideals $m$ of $R$. A local ring is Gorenstein if $\mathrm{id}_{R} R<\infty$, where id $R$ denotes the injective dimension, and an arbitrary commutative noetherian ring $R$ is Gorenstein if $R_{p}$ is Gorenstein for all prime ideals $\underline{p}$ of $R$. For a local ring $R$ a finitely generated module $M$ is Cohen-Macaulay if $\operatorname{depth} M=\operatorname{dim} R$, where $\operatorname{depth} M$ denotes the maximal length of a regular $M$-sequence, and for an arbitrary commutative noetherian ring $R, M$ is Cohen-Macaulay if $M_{\underline{\underline{p}}}$ is a Cohen-Macaulay $R_{\underline{\underline{p}}}$-module for all prime ideals $p$ in $R$.

Let now $\Lambda$ be a noetherian $R$-algebra over an equidimensional Gorenstein ring $R$, i.e., $\Lambda$ is finitely generated as an $R$-module. A $\Lambda$-module $M$ is a lattice if $M$ is a finitely generated Cohen-Macaulay module when considered as an $R$-module and $M_{p}$ is $\Lambda_{\underline{\underline{p}}}$-projective and $\operatorname{Hom}_{R}(M, R)_{\underline{\underline{p}}}$ is $\Lambda_{\underline{\underline{p}}}^{\mathrm{op}}$-projective for all nonmaximal prime ideals $p$ in $R$. Denote by $\mathcal{L}(\Lambda)$ the categöry of lattices. $\Lambda$ is said to be an $R$-order if $\Lambda$ is in $\overline{\mathrm{L}}(\Lambda)$.

We refer to [2] for illustrative examples and basic properties of lattices and orders, and we shall freely use the results from [2] in this paper.

In what follows $R$ will always denote an equidimensional Gorenstein ring of dimension $d$ and $\Lambda$ an $R$-order in the sense just described. noeth $(\Lambda)$ will denote the category of noetherian $\Lambda$-modules, and f.l. $(\Lambda)$ the full subcategory consisting of the $\Lambda$-modules of finite length. Denote by $\left(\mathcal{L}(\Lambda)^{\mathrm{op}}, A b\right)$ the category of contravariant functors from $\mathcal{L}(\Lambda)$ to abelian groups and by f.p. $\left(\mathcal{L}(\Lambda)^{\mathrm{op}}, A b\right)$ the category of 
finitely presented such functors, i.e., $F$ in $\left(\mathscr{L}(\Lambda)^{\mathrm{op}}, A b\right)$ is in f.p. $\left(\mathscr{L}(\Lambda)^{\mathrm{op}}, A b\right)$ if and only if there exist $\Lambda$-lattices $A$ and $B$ and a morphism $f: A \rightarrow B$ such that $(X, A) \rightarrow(X, B) \rightarrow F(X) \rightarrow 0$ is exact for all $X$ in $\mathcal{L}(\Lambda)$.

The main goal of this section is to show that if $F$ is a simple functor in $\left((\mathcal{L}(\Lambda))^{\mathrm{op}}, A b\right)$ not vanishing on projective lattices, then $F$ is finitely presented. This together with [2, Chapter II, Proposition 7.3] then gives that all simple functors in $\left((\mathcal{L}(\Lambda))^{\mathrm{op}}, A b\right)$ are finitely presented. In the next section we will give some applications of this to the case where $R$ is a complete local Gorenstein ring.

Proposition 1.1. Assume $\operatorname{dim} R=1$ and let $M$ be $a \Lambda$-module of finite length. Then there exists an exact sequence $0 \rightarrow L_{1} \rightarrow L_{0} \rightarrow M \rightarrow 0$ with $L_{1}$ and $L_{0}$ lattices and $L_{1}$ an injective lattice. Hence

$$
0 \rightarrow\left(, L_{1}\right)\left|\mathcal{E}(\Lambda) \rightarrow\left(, L_{0}\right)\right| \mathcal{L}(\Lambda) \rightarrow(, M) \mid \mathcal{L}(\Lambda) \rightarrow 0
$$

is exact.

Proof. Assume $M$ is a $\Lambda$-module of finite length. Then the $\Lambda^{\mathrm{op}}$-module $N=$ $\operatorname{Ext}_{R}^{1}(M, R)$ is also of finite length and has the property that $M \simeq \operatorname{Ext}_{R}^{1}(N, R)$. Let $0 \rightarrow K \rightarrow P \rightarrow N \rightarrow 0$ be exact with $P$ a projective $\Lambda^{\mathrm{op}}$-module. Then $K$ is a $\Lambda^{\mathrm{op}}$-lattice and we have the exact sequence

$0=\operatorname{Hom}_{R}(N, R) \rightarrow \operatorname{Hom}_{R}(P, R) \rightarrow \operatorname{Hom}_{R}(K, R) \rightarrow \operatorname{Ext}_{R}^{1}(N, R) \rightarrow \operatorname{Ext}_{R}^{1}(P, R)$.

Since $P$ is a projective lattice $\operatorname{Ext}_{R}^{1}(P, R)=0$ and $\operatorname{Hom}_{R}(P, R)$ is an injective $\Lambda$-lattice. This completes the proof of the proposition.

We now proceed to prove that if $\operatorname{dim} R=d \geqslant 2$ and $A$ is in f.l. $(\Lambda)$, then $(, A) \mid \mathcal{L}(\Lambda)$ is finitely presented.

Proposition 1.2. Let $\Lambda$ be any $R$-order. If $A$ is a $\Lambda$-module of finite length, then there is an exact sequence $L_{1} \rightarrow L_{0} \rightarrow A \rightarrow 0$ with $L_{0}$ and $L_{1}$ lattices such that

$$
\left(, L_{1}\right)\left|\mathcal{L}(\Lambda) \rightarrow\left(, L_{0}\right)\right| \mathcal{L}(\Lambda) \rightarrow(, A) \mid \mathcal{L}(\Lambda) \rightarrow 0
$$

is exact.

Proof. If $\operatorname{dim} R=0$ this is obvious. The case of $\operatorname{dim} R=1$ was shown in Proposition 1.1. So we may assume $\operatorname{dim} R \geqslant 2$. We then have that $\operatorname{Ext}_{R}^{d}(, R)$ is a duality from $\Lambda$-modules of finite length to $\Lambda^{\mathrm{op}}$-modules of finite length with inverse $\operatorname{Ext}_{R}^{d}(, R)$. Now let $A$ be a $\Lambda$-module of finite length and let $B=\operatorname{Ext}_{R}^{d}(A, R)$ which

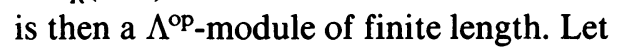

$$
0 \rightarrow \Omega^{d} B \stackrel{t_{d}}{\rightarrow} P_{d-1} \stackrel{t_{d-1}}{\rightarrow} \cdots \rightarrow P_{1} \stackrel{t_{1}}{\rightarrow} P_{0} \stackrel{t_{0}}{\rightarrow} B \rightarrow 0
$$

be a projective resolution of $B$ as a $\Lambda^{\mathrm{op}}$-module. Then the $P_{j}, j=0, \ldots, d-1$, and $\Omega^{d} B$ are in $\mathcal{L}\left(\Lambda^{\mathrm{op}}\right)$ so $\operatorname{Ext}_{R}^{1}\left(P_{j}, R\right)=0=\operatorname{Ext}_{R}^{1}\left(\Omega^{d} B, R\right)$ for $i>0$. This, together with the fact that $\operatorname{Ext}_{R}^{1}(B, R)=0$ for all $i \leqslant d-1$ when $B$ is of finite length, gives the exact sequence

$$
0 \rightarrow P_{0}^{*} \stackrel{t_{1}^{*}}{\rightarrow} P_{1}^{*} \stackrel{t_{2}^{*}}{\rightarrow} P_{2}^{*} \stackrel{t_{3}^{*}}{\rightarrow} \cdots \rightarrow P_{d-1}^{*} \stackrel{t_{d}^{*}}{\rightarrow}\left(\Omega^{d}(B)\right)^{*} \rightarrow \operatorname{Ext}_{R}^{d}(B, R) \rightarrow 0
$$


of $\Lambda$-modules, where $X^{*}=\operatorname{Hom}_{R}(X, R)$. Now $\operatorname{Ext}_{R}^{d}(B, R) \simeq A$ and each $P_{j}^{*}$ is an injective lattice, so $\operatorname{Ext}_{\Lambda}^{i}\left(X, P_{j}^{*}\right)=0$ for all lattices $X$ and $i>0$. This implies that $\operatorname{Ext}^{1}\left(X, U_{i}\right)=0$ for all lattices $X$ and $1 \leqslant i \leqslant d$ where $U_{i}=\operatorname{Im} t_{i}^{*}$. Hence the exactness of the sequence

$$
P_{d-1}^{*} \rightarrow\left(\Omega^{d} B\right)^{*} \rightarrow A \rightarrow 0
$$

implies that $\left(X, P_{d}^{*}\right) \rightarrow\left(X,\left(\Omega^{d} B\right)^{*}\right) \rightarrow(X, A) \rightarrow 0$ is exact for all lattices $X$. Now $\Omega^{d} B$ is a $\Lambda^{\mathrm{op}}$-lattice and therefore $\left(\Omega^{d} B\right)^{*}$ is a $\Lambda$-lattice. We then have that

$$
\left(, P_{d}^{*}\right)\left|\mathcal{L}(\Lambda) \rightarrow\left(,\left(\Omega^{d} B\right)^{*}\right)\right| \mathcal{L}(\Lambda) \rightarrow(, A) \mid \mathcal{L}(\Lambda) \rightarrow 0
$$

is exact and hence the functor $(, A) \mid \mathcal{L}(\Lambda)$ is finitely presented.

We will now use the exact sequence (*) in the proof of Proposition 1.2 to prove the following result.

Proposition 1.3. Let $0 \rightarrow M \rightarrow P \rightarrow P / M \rightarrow 0$ be exact with $P$ projective and $P / M$ of finite length. Then there exist a lattice $L$ and an epimorphism $f: L \rightarrow M$ such that $(, L)|\mathcal{L}(\Lambda) \rightarrow(, M)| \mathcal{L}(\Lambda) \rightarrow 0$ is exact.

Proof. We may assume $\operatorname{dim} R=d \geqslant 2$. Then $(*)$ gives rise to the exact sequence

$$
0 \rightarrow C \rightarrow P_{d-1}^{*} \rightarrow\left(\Omega^{d} B\right)^{*} \rightarrow A \rightarrow 0
$$

where $C=\operatorname{Im} t_{d-1}^{*}$ has the property that $\operatorname{Ext}_{\Lambda}^{1}(X, C)=0$ for all lattices $X$. Let $P \rightarrow\left(\Omega^{d} B\right)^{*} \rightarrow 0$ be exact with $P$ projective. Then we have an exact commutative diagram

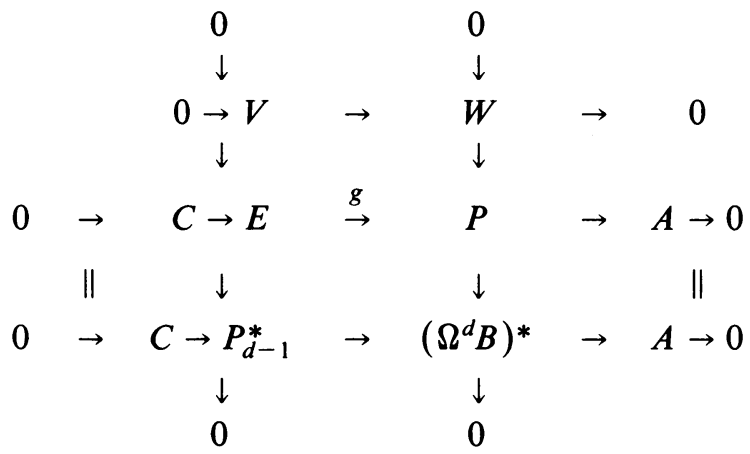

Since $\left(\Omega^{d} B\right)^{*}$ and $P$ are lattices, $W$ is a lattice. Hence $V$ is a lattice because $V \simeq W$. But then $E$ is a lattice since lattices are closed under extensions and $P_{d-1}^{*}$ is a lattice. Therefore the exact sequence $0 \rightarrow C \rightarrow E \rightarrow \operatorname{Im} g \rightarrow 0$ has the property that

$$
0 \rightarrow(, C)|\mathcal{L}(\Lambda) \rightarrow(, E)| \mathcal{L}(\Lambda) \rightarrow(, \operatorname{Im} g) \mid \mathcal{L}(\Lambda) \rightarrow 0
$$

is exact with $E$ a lattice. Now suppose $Q \stackrel{h}{\rightarrow} A \rightarrow 0$ is another epimorphism with $Q$ projective. Then by Schanuel's lemma $Q \amalg I m g \simeq P \amalg K e r h$. Obviously, the exact sequence $0 \rightarrow C \rightarrow E \amalg Q \rightarrow \operatorname{Im} g \amalg Q \rightarrow 0$ also has the property that

$$
0 \rightarrow(, C)|\mathcal{L}(\Lambda) \rightarrow(, E \amalg Q)| \mathcal{L}(\Lambda) \rightarrow(, \operatorname{Im} g \amalg Q) \mid \mathcal{L}(\Lambda) \rightarrow 0
$$

is exact with $E^{1}=E \amalg Q$ a lattice. But now $(, \operatorname{Im} g \amalg Q)|\mathcal{L}(\Lambda) \simeq(, \operatorname{Ker} h \amalg P)| \mathcal{L}(\Lambda)$ and clearly

$$
(, \operatorname{Ker} h \amalg P)|\mathcal{L}(\Lambda) \rightarrow(, \operatorname{Ker} h)| \mathcal{L}(\Lambda) \rightarrow 0
$$


is exact. Therefore the composed morphism $E \amalg Q \rightarrow \operatorname{Ker} h$ also has the property that $(, E \amalg Q)|\mathcal{L}(\Lambda) \rightarrow(, \operatorname{Ker} h)| \mathcal{L}(\Lambda) \rightarrow 0$ is exact. This completes the proof of the proposition.

We are now in a position where we can prove the following theorem.

THEOREM 1.4. Let $S$ be a simple functor in $\left((\mathcal{L}(\Lambda))^{\mathrm{op}}, A b\right)$ not vanishing on projective objects. Then $S$ is finitely presented.

Proof. Since $S$ does not vanish on projectives there is a nonzero projective lattice $P$ such that $S(P) \neq 0$, i.e., we have a morphism $f:(, P) \mid \mathcal{L}(\Lambda) \rightarrow S$ such that $(, P) \mid \mathcal{L}(\Lambda) \rightarrow S \rightarrow 0$ is exact. Ker $f$ is a maximal subfunctor of $(, P) \mid \mathcal{L}(\Lambda)$ and hence is represented by a maximal submodule $M$ of $P$. So $\operatorname{Ker} f=(, M) \mid \mathcal{L}(\Lambda)$ for a maximal submodule $M$ of $P$. But then by Proposition $1.3(, M) \mid \mathcal{L}(\Lambda)$ is finitely generated so we have a lattice $L$ and an epimorphism $g: L \rightarrow M$ such that $\operatorname{Im}(, g)=(, M) \mid \mathcal{L}(\Lambda)=\operatorname{Ker} f$, i.e., we have an exact sequence $(, L) \mid \mathcal{L}(\Lambda) \rightarrow$ $(, P) \mid \mathcal{L}(\Lambda) \rightarrow S \rightarrow 0$ with $P$ and $L$ in $\mathcal{L}(\Lambda)$. This finishes the proof of the theorem.

By duality we have the following result.

THEOREM 1.5. Let $T$ be a simple functor in $(\mathscr{L}(\Lambda), A b)$ not vanishing on injective lattices. Then $T$ is finitely presented.

As a consequence of these theorems, Proposition 7.3 and Proposition 7.5 in Chapter II of [2] we have the following corollary.

COROllary 1.6. (a) Let $S$ be a simple functor in $\left((\mathcal{L}(\Lambda))^{\mathrm{op}}, A b\right)$. Then $S$ is finitely presented. (b) Let $T$ be a simple functor in $(\mathcal{L}(\Lambda), A b)$. Then $T$ is finitely presented.

2. Preprojective partitions. We now want to give some applications of this result in the case $R$ is a complete local noetherian Gorenstein ring of dimension $d$ and $\Lambda$ is an $R$-order. Then $\mathcal{L}(\Lambda)$, the category of $\Lambda$-lattices, is an additive Krull-Schmidt category, i.e., $\ell(\Lambda)$ is an additive category such that each object is a finite direct sum of indecomposable objects, idempotents in $\mathcal{L}(\Lambda)$ split and $\operatorname{End}_{\Lambda}(A)$ is a local ring for all indecomposable objects $A$ in $\mathcal{L}(\Lambda)$. So in the rest of this section we will assume that $R$ is as above. This section is devoted to proving that preprojective partitions exist in this situation.

In order to give the applications we recall some definitions [5]. Let $A$ be an indecomposable object in $\mathcal{L}(\Lambda)$. A morphism $f: A \rightarrow B$ in $\mathcal{L}(\Lambda)$ is said to be left almost split if it is not a splittable monomorphism and whenever there is a nonsplittable monomorphism $g: A \rightarrow X$ in $\mathcal{L}(\Lambda)$, there is an $h: B \rightarrow X$ such that $g=h f$. Dually, a morphism $f: B \rightarrow A$ in $\mathcal{L}(\Lambda)$ is said to be right almost split if it is not a splittable epimorphism and whenever there is a nonsplittable epimorphism $g$ : $X \rightarrow A$ in $\mathcal{L}(\Lambda)$ there is an $h: X \rightarrow B$ such that $g=f h$.

From [2, Chapter II, Proposition 8.1] we have that there is a one-to-one correspondence between the simple functors in $\left((\mathcal{L}(\Lambda))^{\mathrm{op}}, A b\right)$ and the indecomposable objects in $\mathcal{L}(\Lambda)$ given by $A \leftrightarrow(, A) / \underline{r}(, A)$ where $\underline{r}(, A)$ is the unique maximal subfunctor of the representable functor $(, A)$ for an indecomposable lattice $A$. Similarly we have a one-to-one correspondence between the indecomposable objects in $\mathcal{L}(\Lambda)$ and the simple functors in $(\mathscr{L}(\Lambda), A b)$ given by $A \leftrightarrow(A,) / \underline{r}(A$,$) .$ 
We may now restate Corollary 1.6.

Proposition 2.1. For each indecomposable module $A$ in $\mathcal{L}(\Lambda)$ there exists

(i) a left almost split morphism $f: A \rightarrow B$ in $\mathcal{L}(\Lambda)$,

(ii) a right almost split morphism $g: C \rightarrow A$ in $\mathcal{L}(\Lambda)$.

Proof. We only make the observation that $f: A \rightarrow B$ is left almost split if and only if $\operatorname{Coker}(f$,$) is a simple functor in (\mathcal{L}(\Lambda), A b)$. Hence the proposition is just a restatement of Corollary 1.6 in the special case that $R$ is a complete local Gorenstein ring.

By a subcategory $Q$ of $\mathscr{L}(\Lambda)$ we will always mean a full subcategory with the property that if $A \simeq A_{0} \amalg A_{1}$ is in $\mathcal{L}(\Lambda)$ then $A_{0}$ and $A_{1}$ are also in $Q$. If $Q$ is a subcategory of $\mathscr{L}(\Lambda)$, we will let ind $Q$ denote the subcategory of $Q$ consisting of the indecomposable objects in $Q$ and we will let add $Q$ denote the smallest additive subcategory of $\mathscr{L}(\Lambda)$ containing $\mathscr{Q}$. For example, add(ind $\mathcal{L}(\Lambda))=\mathscr{L}(\Lambda)$. A subcategory $\mathcal{Q}$ of $\mathcal{L}(\Lambda)$ will be called a finite subcategory if $Q$ contains only a finite number of nonisomorphic objects, and a subcategory $\mathscr{B}$ of $\mathscr{L}(\Lambda)$ will be said to be of finite type if ind $\mathscr{B}$ is a finite subcategory. We will also use the following conventions. If $A$ is in $\mathscr{L}(\Lambda)$, we will denote by add $A$ the smallest additive subcategory of $\mathscr{L}(\Lambda)$ containing a module isomorphic to $A$. Similarly ind(add $A$ ) will be shortened to ind $A$. From these notions we see that a subcategory $Q$ of $\mathscr{L}(\Lambda)$ is of finite type if and only if ind $\mathcal{Q}=$ ind $A$ for an object $A$ in $\mathcal{L}(\Lambda)$. Similarly, $\mathcal{Q}$ is an additive subcategory of finite type of $\mathcal{L}(\Lambda)$ if and only if $Q=$ add $A$ for an $A$ in $\mathcal{L}(\Lambda)$.

With the radical $\underline{r}_{\mathscr{Q}}($,$) of a subcategory \mathcal{Q}$ of $\mathcal{L}(\Lambda)$, we will mean all morphisms $f$ : $A \rightarrow B$ in $\mathscr{Q}$ such that $p f i \neq 1_{C}$ for all $C$ in $\mathscr{Q}$ and morphisms $i: C \rightarrow A$ and $p: B \rightarrow C$. Since we have assumed that every subcategory is closed under direct summands, we see immediately that $\underline{r}_{\mathbb{Q}}(A, B)=\underline{r}_{\mathcal{L}(\Lambda)}(A, B)$ for all $A$ and $B$ in $Q$. However, when we define power of the radical we have to be careful to indicate to which subcategory we are referring. We define $\underline{r}_{\mathscr{Q}}^{2}(A, B)$ to be the morphisms $f: A \rightarrow B$ in $\mathscr{Q}$ which can be factored as $g: A \rightarrow C$ and $h: C \rightarrow B$ with $C$ in $\mathcal{Q}$ and $g, h$ in $\underline{r}_{\mathfrak{Q}}$. Now $r_{\mathscr{Q}}^{n}(A, B)$ is defined inductively as the morphisms $f: A \rightarrow B$ which can be factored as $g: A \rightarrow C$ and $h: C \rightarrow B$ with $C$ in $\mathcal{Q}, g$ in $\underline{r}_{\mathscr{Q}}^{n-1}(A, C)$ and $h$ in $\underline{r}_{\mathcal{Q}}(C, B)$.

We now want to recall the notion of cover and preprojective partition from [6]. A subcategory $\mathscr{B}$ of a subcategory $\mathscr{Q}$ of $\mathscr{L}(\Lambda)$ is called a cover for $\mathscr{Q}$ if all objects in $Q$ are quotients of a direct sum of objects in $\mathscr{B}$ and a cover is called minimal if no proper subcategory is a cover. For example, let $\underline{\underline{P}}_{0}(\mathcal{L}(\Lambda))=\underline{\underline{P}}_{0}$ be the subcategory of $\mathcal{L}(\Lambda)$ consisting of the indecomposable projective $\Lambda$-lattices. Then it is obvious that $\underline{\underline{P}}_{0}(\mathcal{L}(\Lambda))$ is a cover for $\mathcal{L}(\Lambda)$. Further, it is also easy to see that this is a unique minimal cover, and the objects in $\underline{\underline{P}}_{0}$ satisfy the following property: $A$ is in $\underline{\underline{P}}_{0}$ if and only if whenever there is a surjective morphism $f: B \rightarrow A$ then this morphism is a splittable epimorphism. For an arbitrary subcategory $\mathcal{Q}$ of $\mathcal{L}(\Lambda)$ an object $A$ with the property that each surjective morphism $f: B \rightarrow A$ with $B$ in $\mathcal{Q}$ is a splittable epimorphism will be called a splitting projective object in $Q$. The subcategory of ind $Q$ consisting of the splitting projective objects will be denoted by $\underline{\underline{P}}_{0}(\mathscr{Q})$. Before continuing this discussion, we point out the following connection between minimal 
covers for a subcategory $Q$ of $\mathscr{L}(\Lambda)$ and $\underline{P}_{0}(Q)$, which is a restatement of Theorem 2.3 of $[6]$.

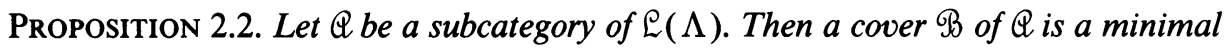
cover if and only if $\mathscr{B}=\underline{\underline{P}}_{0}(\mathbb{Q})$.

We let $\underline{P}_{1}(\mathscr{Q})$ denote the subcategory $\underline{\underline{P}}_{0}\left(\mathscr{Q}_{P_{0}(\mathbb{Q})}\right)$ where $\mathbb{Q}_{\underline{\underline{X}}}$ is the subcategory of $\mathscr{Q}$ consisting of the objects without any direct summand from the subcategory $X$ of $Q$. Now $\underline{\underline{P}}_{n}(\mathscr{Q})$ is defined inductively as $\underline{\underline{P}}_{0}\left(\mathscr{Q}_{\left(\underline{P}_{0}(\mathbb{Q}) \cup \underline{P}_{1}(\mathbb{Q}) \cup \cdots \cup \underline{P}_{n-1}(\mathbb{Q})\right)}\right)$. Finally, we denote the subcategory $\cup_{i<\infty} \underline{P}_{1}(Q)$ of ind $Q$ by $\underline{\underline{P}}(\mathscr{Q})$ and ind $\left(Q_{\underline{P}(\mathbb{Q})}\right)$ by $\underline{\underline{P}}_{\infty}(\mathscr{Q})$. We now list some easily verified properties of these subcategories of $\bar{Q}$.

(a) $\underline{\underline{P}}_{i}(Q) \cap \underline{\underline{P}}_{j}(\mathbb{Q})=\varnothing$ for all $i, j \leqslant \infty$ if $i \neq j$.

(b) If $\underline{\underline{P}}_{i}(Q) \stackrel{=}{=} \varnothing$, then $\underline{\underline{P}}_{j}(\mathbb{Q})=\varnothing$ for $i \leqslant j<\infty$.

In analogy with [6] the collection $\left(\underline{P}_{i}(\mathbb{Q})\right)_{i=0, \ldots, \infty}$ is called a preprojective partition of $Q$ if $\underline{P}_{i}(\mathbb{Q})$ is a finite cover for $\mathbb{Q}_{\underline{P}_{0}(\mathbb{Q}) \cup \cdots \cup \underline{P}_{i-1}(\mathbb{Q})}$ for each $i<\infty$.

We say that $Q$ has a preprojective partition if $\left\{\underline{\underline{P}}_{i}(\mathbb{Q})\right\}_{i=0, \ldots, \infty}$ is a preprojective partition of $Q$. Finally, if $Q$ has a preprojective partition, then we say that $A$ in $Q$ is preprojective if every indecomposable summand of $A$ is in $\underline{\underline{P}}(\mathbb{Q})=\cup_{i<\infty} \underline{\underline{P}}_{i}(\mathbb{Q})$.

We will use the duality $\operatorname{Hom}_{R}(, R)=D: \mathcal{L}(\Lambda) \rightarrow \mathcal{L}\left(\overline{\overline{\Lambda^{\mathrm{op}}}}\right)$ to introduce the dual notion of cocover and preinjective partition. Let $\mathcal{Q}$ be a subcategory of $\mathscr{L}(\Lambda)$. A subcategory $\mathscr{B}$ of $Q$ is called a cocover for $Q$ if $D \mathscr{B}$ is a cover for $D Q$ in $\mathscr{L}\left(\Lambda^{\text {op }}\right)$. A cocover is minimal if no proper subcategory is a cocover. Splitting injective objects and preinjective partitions are also introduced using this duality, but since this is straightforward we leave it to the reader to write down the formal definitions.

As for artin algebras [6, Theorem 3.1] we have the following result for lattices.

TheOREM 2.3. Let $\mathcal{C}$ be a subcategory of $\mathcal{L}(\Lambda)$ of finite type. Then $\mathcal{C}$ has both $a$ preprojective and a preinjective partition.

Before we go on to prove the existence of a preprojective partition of $\mathcal{L}(\Lambda)$ and other subcategories $\mathscr{Q}$ of $\mathcal{L}(\Lambda)$ we will give some more information about the relation between $\underline{\underline{P}}_{j}(\mathbb{Q})$ and $\cup_{i<j} \underline{\underline{P}}_{i}(\mathbb{Q})(j<\infty)$, when $\mathscr{Q}$ is a subcategory of $\mathcal{L}(\Lambda)$ having a preprojective partition. To do that we start out with the following observation.

LEMMA 2.4. Let $Q$ be a subcategory of $\mathcal{L}(\Lambda)$ of finite type. Then there exists an $n$ such that whenever $f: A \rightarrow B$ in $Q$ is in $\underline{r}_{\mathbb{Q}}^{n}(A, B)$, then $f(A) \subseteq \underline{r}_{\Lambda} B$, where $\underline{r}_{\Lambda}$ is the radical of $\Lambda$.

Proof. Let $A_{1}, \ldots, A_{k}$ be a complete set of nonisomorphic indecomposable modules in $\mathcal{Q}$, let $A=A_{1} \amalg \cdots \amalg A_{k}$ and consider $\operatorname{End}_{\Lambda}(A)$. By fixing an isomorphism $g_{M_{i}}: M_{i} \rightarrow A_{i}$ for each module $M_{i}$ isomorphic to $A_{i}$ in ind(Q), we may now embed $\operatorname{ind}(Q)$ in $\operatorname{End}(A)$ according to the matrix representation of $\operatorname{End}(A)$ as $\left(\operatorname{Hom}\left(A_{i}, A_{j}\right)\right)_{i, j}$. By this embedding the radical of $\operatorname{ind}(\mathbb{Q})$ goes into the radical of $\operatorname{End}(A) . A$ and $\underline{r}_{\Lambda}(A)$ are both $\operatorname{End}(A)$-modules in a natural way. Therefore the $\Lambda$-module $A / \underline{r}_{\Lambda}(A)$ of finite length is an $\operatorname{End}(A)$-module. Since $A / \underline{r}_{\Lambda}(A)$ also has finite length as an $R$-module, it is also of finite length as an $\operatorname{End}(A)$-module. But then $A / \underline{r}_{\Lambda}(A)$ is annihilated by some power $n$ of the radical of $\operatorname{End}(A)$. That means 
that each $f: A^{\prime} \rightarrow A^{\prime \prime}$ in $\underline{r}_{\text {ind }(\mathcal{Q})}^{n}$ maps $A^{\prime}$ to $\underline{r}_{\Lambda} A^{\prime \prime}$ and hence if $f: B^{\prime} \rightarrow B^{\prime \prime}$ is in $\underline{r}_{\mathscr{Q}}^{n}$ then $f\left(B^{\prime}\right) \subseteq \underline{r}_{\Lambda} B^{\prime \prime}$, which completes the proof of the lemma.

Proposition 2.5. Let $Q$ be a subcategory of $\mathscr{L}(\Lambda)$ having a preprojective partition $\underline{\underline{P}}_{0}(\mathbb{Q}), \underline{\underline{P}}_{1}(\mathbb{Q}), \ldots, \underline{\underline{P}}_{n}(\mathbb{Q}), \ldots, \underline{\underline{P}}_{\infty}(\mathbb{Q})$. Let $A$ be a nonzero object in $\underline{\underline{P}}_{j}(\mathbb{Q}), 0<j<\infty$. Then there exist a $B$ in $\cup_{i<j} \underline{P}_{i}(\mathbb{Q})$ and a morphism $f: B \rightarrow A$ not in $\underline{r}_{Q}^{2}$.

Proof. Let $A$ be in $\underline{P}_{j}(Q)$ with $0<j<\infty$ and assume that $\operatorname{Hom}_{\Lambda}(B, A) \subseteq$ $\underline{r}_{\mathscr{Q}}^{2}(B, A)$ for all $B$ in $\cup_{i<j} \underline{P}_{i}(\mathscr{Q})$. Now since $\cup_{i<j} \underline{P}_{i}(\mathbb{Q})$ is a cover for $\mathbb{Q}$ there exist a $B$ in add $\cup_{i<j} \underline{P}_{i}(Q)$ and a surjective morphism $f: B \rightarrow A$. By assumption $f$ is in $\underline{r}_{\mathscr{Q}}^{2}$ so we may factor $f$ as $B \rightarrow^{f^{1}} B^{1} \rightarrow^{g^{1}} A$ with $f^{1}$ and $g^{1}$ in $\underline{r}_{Q}$. Now decompose $B^{1}$ as $B_{1} \amalg C_{1}$ with $B_{1}$ in add $\cup_{i<j} \underline{P}_{i}(\mathscr{Q})$ and $C_{1}$ in $\mathcal{Q}_{\cup_{i<j} \underline{P}_{i}(\mathscr{Q})}$, write $g^{1}=\left(g_{1}, h_{1}\right)$ and $f^{1}=\left(\begin{array}{l}f_{1} \\ k_{1}\end{array}\right)$. Then $f_{1}, k_{1}, g_{1}$ and $h_{1}$ are in $\underline{r}_{\mathfrak{Q}}$ and by assumption $g_{1}$ is in $r_{\mathbb{Q}}^{2}$. Now by an easy induction argument we see that for each natural number $n$ we can factor $f$ : $B \rightarrow A$ as

$$
B \stackrel{\left(f_{n}^{n}\right)}{\rightarrow} B_{n} \amalg C_{n} \stackrel{\left(g_{n}, h_{n}\right)}{\rightarrow} A
$$

with $g_{n} f_{n}$ in $\underline{r}_{Q}^{n+2}, h_{n}: C_{n} \rightarrow A$ in $\underline{r}_{\mathcal{Q}}$ and $C_{n}$ in $\mathcal{Q}_{\cup_{i<j} P_{i}(\mathcal{Q})}$. By Lemma $2.4 \operatorname{Im} g_{n} f_{n} \subseteq \underline{r}_{\Lambda} A$ for some $n$ since $\cup_{i<j} \underline{P}_{i}(\mathscr{Q})$ is a subcategory of $\mathscr{Q}$ of finite type. So $\operatorname{Im} h_{n} k_{n}=A$ by Nakayama's lemma. But then $h_{n}: C_{n} \rightarrow A$ is a surjective morphism in $\mathbb{Q}_{\cup_{i<j} P_{i}(\mathcal{Q})}$ which is not a splittable surjection. This is a contradiction to $A$ being a splitting projective object in $\mathbb{Q}_{\cup_{i<j} \underline{P}_{i}(\mathscr{Q})}$ which completes the proof of the proposition.

We will now prove the existence of the preprojective partition of $\mathscr{L}(\Lambda)$. To do this we need the following analogue of [6, Lemma 3.14.b].

Lemma 2.6. Suppose $\varrho \supseteq D$ are subcategories of $\mathcal{L}(\Lambda)$. Assume $\mathcal{C}$ has left almost split morphisms. Then for each $C$ in $\bigcup_{D}$ and integer $n \geqslant 0$, there is a morphism $g$ : $C \rightarrow D \amalg Z$ with $D$ in add $D$ and $Z$ in add $\bigodot_{D}$ such that pg is in $\underline{r}_{D}^{n}(C, Z)$ and $\operatorname{Im}(g, X)=(C, X)$ for all $X$ in $D$, where $p: D \amalg Z \rightarrow Z$ is the projection morphism.

Proof. The proof of this is exactly the same as in the artin algebra case, so we just refer to [6, Lemma 3.14].

By combining the results of Proposition 2.1, Lemma 2.4 and Lemma 2.6, we have:

Proposition 2.7. Let $\mathcal{Q}$ be a subcategory of finite type of $\mathcal{L}(\Lambda)$. Then $\mathcal{L}(\Lambda)_{\mathfrak{Q}}$ has a finite cover.

Proof. Let $\mathcal{Q}$ be a subcategory of finite type of $\mathcal{L}(\Lambda)$. By Lemma 2.4 we then know that there exists a natural number $m$ such that whenever $f: B \rightarrow A$ is in $\underline{r}_{\mathscr{Q}}^{m}$ then $f(B) \subseteq \underline{r}_{\Lambda}(A)$. By Proposition $2.1 \mathcal{L}(\Lambda)$ has left almost split morphisms so using Lemma 2.6 with $\mathcal{C}=\mathcal{L}(\Lambda), D=\mathcal{L}(\Lambda)_{\mathscr{Q}}$ and $m=n$ we get for each projective lattice $P$ in add $Q=\mathcal{C}_{D}$ a morphism $g: P \rightarrow D_{P} \amalg Z$ with $D_{P}$ in $\mathcal{L}(\Lambda)_{\mathscr{Q}}, Z$ in add $\mathcal{Q}, p g$ in $\underline{r}_{\mathscr{Q}}^{m}$ and $\operatorname{Im}(g, X)=(P, X)$ for all $X$ in $\mathcal{L}(\Lambda)_{\mathfrak{Q}}$. Now let $\Re$ be the subcategory of $\mathscr{L}(\Lambda)_{\mathfrak{Q}}$ consisting of all indecomposable projective lattices not in $Q$ and of all indecomposable summands in $D_{P}$ where $P$ is a direct sum of all indecomposable projective lattices in $\mathcal{Q}$. It is now obvious that $\mathscr{B}$ is a cover for $\mathcal{L}(\Lambda)_{\mathfrak{Q}}$.

As a trivial consequence of this, we have the existence of the preprojective partition for all subcategories $\mathscr{L}(\Lambda)_{\mathbb{Q}}$ of $\mathscr{L}(\Lambda)$ when $\mathbb{Q}$ is a subcategory of finite type. 
THEOREM 2.8. $\mathscr{L}(\Lambda)$ and all subcategories $\mathscr{L}(\Lambda)_{\mathfrak{Q}}$ of $\mathscr{L}(\Lambda)$ have a preprojective partition when $Q$ is a subcategory of finite type.

3. Finitely presented functors. We will now go back to the general case again where $R$ is an arbitrary equidimensional Gorenstein ring and $\Lambda$ is an $R$-order. Let $\mathcal{C}(\Lambda)$ denote the full subcategory of noeth $\Lambda$ consisting of the modules $M$ such that $M_{p}$ is $\Lambda_{p}$-projective for all nonmaximal prime ideals $p$ of $R$. It is obvious that $\mathcal{L}(\Lambda)$ and the category f.l. $(\Lambda)$ are both subcategories in $C(\Lambda)$. In $\S 1$ we proved that all modules $A$ of finite length in $\mathcal{C}(\Lambda)$ had the property that the functor $(, A) \mid \mathcal{L}(\Lambda)$ is finitely presented. The aim of this section is to show that the same is true for all of $\mathcal{C}(\Lambda)$, i.e., for each $B$ in $\mathcal{C}(\Lambda),(, B) \mid \mathcal{E}(\Lambda)$ is finitely presented. To do this we first make the following reduction.

Proposition 3.1. If for each $M$ in $\mathcal{C}(\Lambda)$ we have that $(, M) \mid \mathcal{L}(\Lambda)$ is finitely generated, then for each $M$ in $\mathcal{C}(\Lambda),(, M) \mid \mathcal{L}(\Lambda)$ is in f.p. $\left(\mathcal{L}(\Lambda)^{\mathrm{op}}\right.$, Ab) and has $a$ projective resolution in f.p. $\left(\mathcal{L}(\Lambda)^{\mathrm{op}}, A b\right)$.

Proof. Assume $M$ is in $C(\Lambda)$. Then by hypothesis we have that there is an epimorphism $L \rightarrow^{f} M \rightarrow 0$ with $L$ a lattice such that $(, L)|\mathcal{L}(\Lambda) \rightarrow(, M)| \mathscr{L}(\Lambda) \rightarrow 0$ is exact. But the exactness of the sequence $0 \rightarrow \operatorname{Ker} f \rightarrow L \rightarrow M \rightarrow 0$ implies that $\operatorname{Ker} f$ is in $C(\Lambda)$. From this the proposition follows trivially.

We will now increase the family of modules $M$ in $C(\Lambda)$ such that $(, M) \mid \mathcal{L}(\Lambda)$ is finitely generated to contain all reflexive modules. To do this we have to go through another subcategory $g(\Lambda)$ of $e(\Lambda)$ which we will now define. (For details see [2].) Assume $\operatorname{dim} R=d$. Let $g(\Lambda)$ denote the full subcategory of $C(\Lambda)$ consisting of those modules $M$ such that $\operatorname{Ext}_{\Lambda}^{i}(M, \Lambda)=0$ for all $i=1, \ldots, d$. As an intermediate result we have the following.

THEOREM 3.2. For each $M$ in $\mathcal{C}(\Lambda)$ there is an epimorphism $J \rightarrow M$ with $J$ in $\mathscr{G}(\Lambda)$ such that $(X, J) \rightarrow(X, M) \rightarrow 0$ is exact for all $X$ in $\mathscr{G}(\Lambda)$, i.e., $(, M) \mid \mathcal{G}(\Lambda)$ is finitely generated.

This theorem will follow from the following. We first recall that $\operatorname{Hom}_{\Lambda}(A, B)$ denotes the factor group of $\operatorname{Hom}_{\Lambda}(A, B)$ obtained by dividing out the subgroup consisting of those homomorphisms factoring through a projective module.

Proposition 3.3. Let $M$ be in $\mathrm{C}(\Lambda)$. Then there is a morphism $J \rightarrow M$ with $J$ in $\mathcal{G}(\Lambda)$ such that the induced morphism $\operatorname{Hom}_{\Lambda}(X, J) \rightarrow \operatorname{Hom}_{\Lambda}(X, M)$ is an isomorphism for all $X$ in $\mathscr{G}(\Lambda)$.

Proof. Let $M$ be in $\mathcal{C}(\Lambda)$ and let $0 \rightarrow \Omega^{d}(M) \rightarrow P_{d-1} \rightarrow \cdots \rightarrow P_{1} \rightarrow P_{0} \rightarrow M \rightarrow 0$ be exact where $d=\operatorname{dim} R$. Then $\Omega^{d}(M)$ is a lattice [2]. By [2, Chapter I, Theorem 7.8] $\Omega^{d}: \mathscr{g}(\Lambda) \rightarrow \mathscr{L}(\Lambda)$ is an equivalence where $\mathscr{g}(\Lambda)$ and $\mathscr{L}(\Lambda)$ are the categories modulo projectives. Hence there is a $J$ in $\mathscr{f}(\Lambda)$ such that $\Omega^{d}(J) \simeq \Omega^{d} M$. Now since $X$ in $\mathcal{G}(\Lambda)$ has the property that $\operatorname{Ext}^{i}(X, \Lambda)=0$ for $i=1, \ldots, d$, it follows that the functor $\Omega^{d}$ : noeth $\Lambda \rightarrow$ noeth $\Lambda$ has the property $\Omega^{d}: \operatorname{Hom}(X, Y) \rightarrow \operatorname{Hom}\left(\Omega^{d} X, \Omega^{d} Y\right)$ 
is an isomorphism for all $X$ in $g(\Lambda)$ and all $Y$ in noeth $\Lambda$. (See [3, p. 88].) Hence $\Omega^{d}$ : Hom $M) \simeq \operatorname{Hom}\left(\Omega^{d} J, \Omega^{d} M\right)$. So there is a morphism $f: J \rightarrow M$ such that $\Omega^{d}(f)$ : $\overline{\Omega^{d} J} \rightarrow \Omega^{d} M$ is our given isomorphism. Suppose now that $X$ is in $g(\Lambda)$. Then we have the commutative diagram

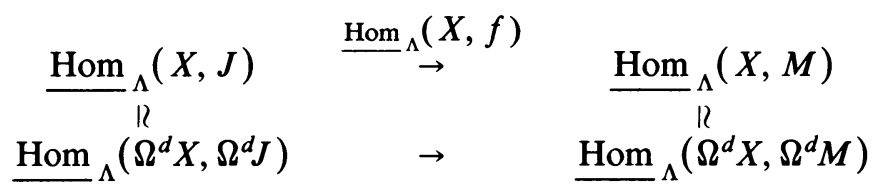

Hence $\operatorname{Hom}_{\Lambda}(X, f): \operatorname{Hom}_{\Lambda}(X, J) \rightarrow \operatorname{Hom}_{\Lambda}(X, M)$ is an isomorphism for all $X$ in $g(\Lambda)$. This completes the proof of the proposition.

We can now prove Theorem 3.2. Let $M$ be in $\mathcal{C}(\Lambda)$. Then by Proposition 3.3 there exist a $J$ in $g(\Lambda)$ and a homomorphism $f: J \rightarrow M$ such that $(, f):(, J) \mid g(\Lambda) \rightarrow$ $(, \underline{M}) \mid g(\Lambda)$ is an isomorphism. Let $P$ be a projective module in noeth $\Lambda$ and $u$ : $P \rightarrow M$ an epimorphism. Then $P$ is in $g(\Lambda)$. Let $t: P \amalg J \rightarrow M$ be the morphism induced by $f$ and $u$. Let $h: X \rightarrow M$ be a morphism with $X$ in $g(\Lambda)$. Then $\underline{h} \in(X, M)$ is equal to $f g$ for some $g: X \rightarrow J$ since $f$ is an isomorphism. But then $\bar{h}-\overline{f g \in P(X, M)}$, the subgroup of $(X, M)$ consisting of all morphisms factoring through a projective module. Thus $h-f g=u s$ for some $s: X \rightarrow P$. Hence $h=u s+f g$ which shows that $h \in \operatorname{Im}(X, t)$. This shows that

$$
(X, P \amalg J) \stackrel{(X, t)}{\rightarrow}(X, M) \rightarrow 0
$$

is exact for all $X$ in $g(\Lambda)$ and so $(, P \amalg J)|g(\Lambda) \rightarrow(, M)| g(\Lambda) \rightarrow 0$ is exact. Hence $(, M) \mid \mathcal{G}(\Lambda)$ is finitely generated.

In the case $\Lambda$ is a Gorenstein $R$-order, i.e., $\Lambda$ is an $R$-order such that $\Lambda \simeq$ $\operatorname{Hom}_{R}(\Lambda, R)$ as a two-sided $\Lambda$-module, we can use this result to obtain the following, which is our main result in this situation.

Proposition 3.4. Let $\Lambda$ be a Gorenstein $R$-order. For each $M$ in $C(\Lambda)$, there is an epimorphism $L \rightarrow M$ with $L$ a lattice such that $(, L)|\mathcal{L}(\Lambda) \rightarrow(, M)| \mathcal{L}(\Lambda) \rightarrow 0$ is exact.

Proof. Assume $\Lambda$ is a Gorenstein $R$-order. Then $g(\Lambda)=\mathcal{L}(\Lambda)$ by [2, Chapter III, Theorem 1.1] and the result follows from Theorem 3.2.

As another consequence of Proposition 3.3 we have the following covariant version of the original problem which will be used to prove that $(, M) \mid \mathscr{L}(\Lambda)$ is finitely generated for all reflexive modules in $C(\Lambda)$.

Proposition 3.5. Let $M$ be in $C(\Lambda)$ where $\Lambda$ is an arbitrary $R$-order. Then there is a lattice $L$ and a morphism $f: M \rightarrow L$ such that $(L),\left|\mathcal{L}(\Lambda) \rightarrow^{(f,)}(M),\right| \mathcal{L}(\Lambda) \rightarrow 0$ is exact.

Proof. Let $M$ be in $\mathcal{C}(\Lambda)$. Then $\operatorname{Tr} M$ is in $\mathcal{C}\left(\Lambda^{\mathrm{op}}\right)$. By Proposition 3.3 we know there is a $J$ in $\mathcal{G}\left(\Lambda^{\text {op }}\right)$ and a morphism $f: J \rightarrow \operatorname{Tr} M$ such that

$$
(, \underline{J})\left|\mathcal{L}\left(\Lambda^{\mathrm{op}}\right) \stackrel{(, f)}{\rightarrow}(, \underline{\operatorname{Tr} M})\right| \mathcal{g}\left(\Lambda^{\mathrm{op}}\right)
$$


is an isomorphism. By [2, Chapter I, Theorem 7.9] $\mathrm{Tr}$ induces an equivalence $\mathrm{Tr}$ : $\mathcal{L}(\Lambda) \rightarrow g\left(\Lambda^{\text {op }}\right)$. Therefore, by choosing a representative $h$ for $\operatorname{Tr} f$ in $(M, \operatorname{Tr} J)$ we have a morphism $h: M \rightarrow \operatorname{Tr} J$ such that $(\operatorname{Tr} J),|\mathcal{L}(\Lambda) \rightarrow \stackrel{(h,)}{ }(\underline{\bar{M}},-)| \mathcal{L}(\Lambda)$ is an isomorphism. Since $(M, \Lambda)$ is finitely generated as an $R$-module, there exist a projective module $Q$ and a morphism $t: M \rightarrow Q$ such that $(Q, P) \rightarrow(M, P) \rightarrow 0$ is exact for all projective modules $P$. Since $(\underline{h}$,$) is an isomorphism, it is now easy$ to see the morphism $M \rightarrow^{(t, h)} Q \amalg \operatorname{Tr} J$ induces a surjection $(Q \amalg \operatorname{Tr} J) \mid, \mathcal{L}(\Lambda) \rightarrow$ $(M) \mid, \mathcal{L}(\Lambda)$.

As a consequence of this proposition we have:

COROLlary 3.6. Let $\Lambda$ be an arbitrary $R$-order. Suppose $M$ is in $\mathcal{C}(\Lambda)$ such that $M$ is reflexive over $R$. Then there is a lattice $L$ and an epimorphism $L \rightarrow M$ such that $(, L)|\mathcal{L}(\Lambda) \rightarrow(, M)| \mathcal{L}(\Lambda) \rightarrow 0$ is exact.

Proof. Let $M$ in $\mathcal{C}(\Lambda)$ be reflexive. Then $\operatorname{Hom}_{R}(M, R)$ is in $\mathcal{C}\left(\Lambda^{\mathrm{op}}\right)$ and therefore there exist a $\Lambda^{\text {op }}$-lattice $L$ and a morphism $f: \operatorname{Hom}_{R}(M, R) \rightarrow L$ such that $(L),\left|\mathcal{L}\left(\Lambda^{\mathrm{op}}\right) \rightarrow\left(\operatorname{Hom}_{R}(M, R),\right)\right| \mathcal{L}\left(\Lambda^{\mathrm{op}}\right) \rightarrow 0$ is exact. Since $M, \operatorname{Hom}_{R}(M, R)$ and lattices are $R$-reflexive, we get that

$$
\left(, \operatorname{Hom}_{R}(L, R)\right)|\mathcal{L}(\Lambda) \stackrel{(,(f, R))}{\rightarrow}(, M)| \mathcal{L}(\Lambda) \rightarrow 0 \text { is exact. }
$$

We are now able to extend the class of modules $M$ in $\mathcal{C}(\Lambda)$ such that $(, M) \mid \mathcal{L}(\Lambda)$ is finitely generated to contain the class of torsionless modules in $e(\Lambda)$.

PROPOSITION 3.7. Let $\Lambda$ be an arbitrary $R$-order and let $M$ be a torsionless module in $\mathcal{C}(\Lambda)$. Then $(, M) \mid \mathcal{L}(\Lambda)$ is finitely generated.

Proof. Let $M$ be torsionless in $C(\Lambda)$. Then we have an exact sequence $0 \rightarrow M \rightarrow$ $M^{* *} \rightarrow U \rightarrow 0$ where $U$ is of finite length since $M$ is in $C(\Lambda)$. Therefore by Proposition 1.3 there is an exact sequence $0 \rightarrow K \rightarrow L \rightarrow U \rightarrow 0$ with $L$ a lattice such that $0 \rightarrow(, K)|\mathcal{L}(\Lambda) \rightarrow(, L)| \mathcal{L}(\Lambda) \rightarrow(, U) \mid \mathcal{L}(\Lambda) \rightarrow 0$ is exact with $(, K) \mid \mathcal{L}(\Lambda)$ finitely generated. From this we obtain the exact commutative diagram

$$
\begin{aligned}
& \begin{array}{cc}
0 & 0 \\
\downarrow & \downarrow
\end{array} \\
& (, K)|\mathfrak{L}(\Lambda)=(, K)| \mathfrak{L}(\Lambda) \\
& 0 \rightarrow(, M)\left|\mathfrak{L}(\Lambda) \rightarrow\left(, M^{* *} x_{U} L\right)\right| \mathfrak{L}(\Lambda) \rightarrow(, L)\left|\mathfrak{L}(\Lambda) \rightarrow \operatorname{Ext}^{1}(, M)\right| \mathfrak{L}(\dot{\Lambda}) \\
& 0 \rightarrow(, M)\left|\mathfrak{L}(\Lambda) \rightarrow\left(, M^{* *}\right)\right| \mathfrak{L}(\Lambda) \rightarrow(, U)\left|\mathfrak{L}(\Lambda) \rightarrow \operatorname{Ext}^{\prime}(, M)\right| \mathfrak{L}(\Lambda) \\
& \downarrow
\end{aligned}
$$

By the five lemma $0 \rightarrow(, K)\left|\mathfrak{L}(\Lambda) \rightarrow\left(, M^{* *} x_{U} L\right)\right| \mathfrak{L}(\Lambda) \rightarrow\left(, M^{* *}\right) \mid \mathfrak{L}(\Lambda) \rightarrow 0$ is exact. But $(, K) \mid \mathcal{L}(\Lambda)$ is finitely generated by Proposition 1.2 and by Corollary 3.6 $\left(, M^{* *}\right) \mid \mathcal{L}(\Lambda)$ is finitely generated. Therefore, also $\left(, M^{* *} x_{U} L\right) \mid \mathcal{L}(\Lambda)$ is finitely generated since finitely generated functors are closed under extensions. Therefore 
there exist a lattice $L_{0}$ and an epimorphism $L_{0} \rightarrow M^{* *} x_{U} L$ such that $\left(, L_{0}\right) \mid \mathcal{L}(\Lambda)$ $\rightarrow\left(, M^{* *} x_{U} L\right) \mid \mathcal{L}(\Lambda) \rightarrow U$ is exact. This gives rise to the commuting diagram

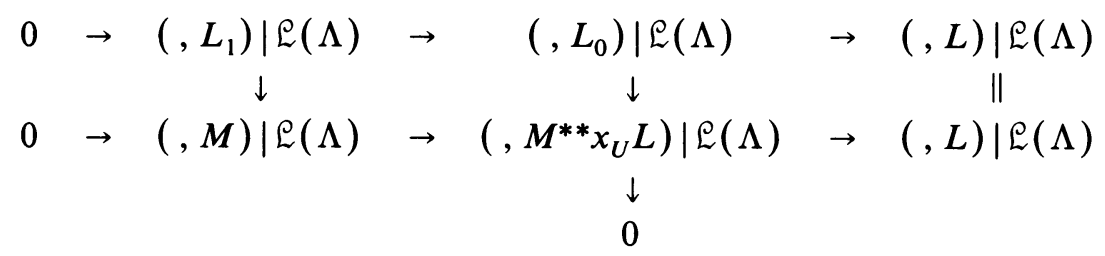

which again by the five lemma implies that the morphism $L_{1} \rightarrow M$ has the property that $\left(, L_{1}\right)|\mathcal{L}(\Lambda) \rightarrow(, M)| \mathcal{L}(\Lambda) \rightarrow 0$ is exact.

To complete the proof of our main result we now only have to consider modules $M$ in $C(\Lambda)$ such that $\operatorname{Ker} f$ is nontrivial where $f: M \rightarrow M^{* *}$ is the usual evaluation map. To do this we need the following general result.

Proposition 3.8. Let $\Lambda$ be an $R$-order and let $0 \rightarrow R \rightarrow I_{0} \rightarrow \cdots \rightarrow I_{d} \rightarrow 0$ be $a$ minimal injective resolution of $R$. For each $X$ in $\mathcal{C}(\Lambda)$ we have that

$$
\operatorname{Ext}_{\Lambda}^{1}\left(, \operatorname{Hom}_{R}\left(X, I_{d}\right)\right)\left|\mathcal{L}(\Lambda) \simeq \operatorname{Ext}_{\Lambda}^{1}\left(, \operatorname{Hom}_{R}\left(\Omega^{d} X, R\right)\right)\right| \mathcal{L}(\Lambda) .
$$

Proof. Let $X$ be in $\mathcal{C}\left(\Lambda^{\text {op }}\right)$ and $0 \rightarrow \Omega^{d} X \rightarrow P_{d-1} \rightarrow \cdots \rightarrow P_{1} \rightarrow P_{0} \rightarrow X \rightarrow 0$ be an exact sequence of $\Lambda^{\mathrm{op}}$-modules with $P_{j}$ projective and $d=\operatorname{dim} R$. Then we have

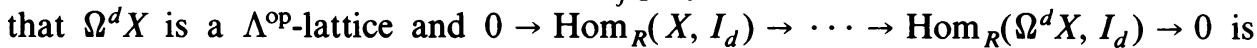
exact when $I_{d}$ is the last term in an injective resolution of $R$ over $R$. Moreover each

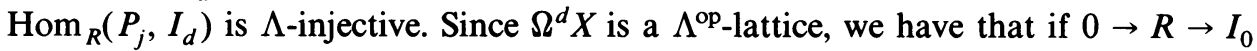
$\rightarrow \cdots \rightarrow I_{d} \rightarrow 0$ is a minimal injective resolution, then $0 \rightarrow \operatorname{Hom}_{R}\left(\Omega^{d} X, R\right) \rightarrow \cdots \rightarrow$ $\operatorname{Hom}_{R}\left(\Omega^{d} X, I_{d}\right) \rightarrow 0$ is exact [2]. Further, since $\Omega^{d} X$ is in $\mathcal{C}(\Lambda)$, $\operatorname{Tor}_{1}^{\Lambda}\left(A, \Omega^{d} X\right)_{p}$ are zero for all $A$ in noeth $\Lambda$ and nonmaximal prime ideals $p$ of $R$. But then $\operatorname{Hom}_{R}\left(\operatorname{Tor}_{1}^{\Lambda}\left(A, \Omega^{d} X\right), I_{j}\right)=0$ for all $0 \leqslant j \leqslant d-1$. Then since $I_{j}$ is an injective $R$-module

$$
\operatorname{Hom}_{R}\left(\operatorname{Tor}_{1}^{\Lambda}\left(A, \Omega^{d} X\right), I_{j}\right) \simeq \operatorname{Ext}_{\Lambda}^{1}\left(A, \operatorname{Hom}_{R}\left(\Omega^{d} X, I_{j}\right)\right)
$$

[8, p. 120], which shows that $\operatorname{Hom}_{R}\left(\Omega^{d} X, X_{j}\right)$ is an injective $\Lambda$-module for $0 \leqslant j \leqslant d$ - 1. Therefore,

$\operatorname{Ext}_{\Lambda}^{d+1}\left(, \operatorname{Hom}_{R}\left(X, I_{d}\right)\right) \simeq \operatorname{Ext}_{\Lambda}^{1}\left(, \operatorname{Hom}_{R}\left(\Omega^{d} X, I_{d}\right)\right) \simeq \operatorname{Ext}_{\Lambda}^{d+1}\left(, \operatorname{Hom}_{R}\left(\Omega^{d} X, R\right)\right)$.

From [2, Chapter I, Proposition 7] we have that $\Omega^{d}: \underline{\mathscr{f}(\Lambda)} \rightarrow \underline{\mathcal{L}(\Lambda)}$ is an equivalence. So

$$
\begin{aligned}
\operatorname{Ext}_{\Lambda}^{1}\left(, \operatorname{Hom}_{R}\left(X, I_{d}\right)\right)\left|\mathcal{L}(\Lambda) \simeq \operatorname{Ext}_{\Lambda}^{1}\left(\Omega^{d}, \operatorname{Hom}_{R}\left(X, I_{d}\right)\right)\right| g(\Lambda) \\
\simeq \operatorname{Ext}_{\Lambda}^{d+1}\left(, \operatorname{Hom}_{R}\left(X, I_{d}\right)\right)\left|\mathcal{g}(\Lambda) \simeq \operatorname{Ext}_{\Lambda}^{d+1}\left(, \operatorname{Hom}_{R}\left(\Omega^{d} X, R\right)\right)\right| \mathcal{g}(\Lambda) \\
\simeq \operatorname{Ext}_{\Lambda}^{1}\left(, \operatorname{Hom}_{R}\left(\Omega^{d} X, R\right)\right) \mid \mathcal{E}(\Lambda) .
\end{aligned}
$$

We are now able to prove our promised result.

THEOREM 3.9. For each module $A$ in $\mathcal{C}(\Lambda)$ the functor $(, A) \mid \mathcal{L}(\Lambda)$ is finitely presented. 
Proof. Let $M$ be in $\mathcal{C}(\Lambda)$. If $M$ is torsionless, we have that $(, M) \mid \mathcal{L}(\Lambda)$ is finitely generated by Proposition 3.7, so we may assume $M$ is not torsionless. Then $\operatorname{Ker}\left(M \rightarrow M^{* *}\right)$ has finite length so we have an exact sequence $0 \rightarrow U \rightarrow M \rightarrow M^{\prime \prime} \rightarrow$ 0 with $U$ of finite length and $M^{\prime \prime}$ torsionless. Since $M^{\prime \prime}$ is torsionless and in $\mathcal{C}(\Lambda)$, there is by Proposition 3.7 a map $L \rightarrow M^{\prime \prime}$ where $L$ is a lattice such that $(, L)\left|\mathcal{L}(\Lambda) \rightarrow\left(, M^{\prime \prime}\right)\right| \mathcal{L}(\Lambda) \rightarrow 0$ is exact. Letting

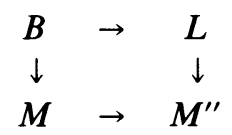

be a pullback diagram, we have the following exact commutative diagram

$$
\begin{aligned}
& 0 \rightarrow(, U)|\mathfrak{L}(\Lambda) \rightarrow(, B)| \mathfrak{L}(\Lambda) \rightarrow \underset{\downarrow}{\|} \rightarrow(, L)\left|\mathfrak{L}(\Lambda) \rightarrow \operatorname{Ext}^{1}(, U)\right| \mathfrak{L}(\Lambda) \\
& 0 \rightarrow(, U)|\mathfrak{L}(\Lambda) \rightarrow(, M)| \mathfrak{L}(\Lambda) \rightarrow\left(, M^{\prime \prime}\right)\left|\mathfrak{L}(\Lambda) \rightarrow \operatorname{Ext}^{1}(, U)\right| \mathfrak{L}(\Lambda) \\
& \downarrow
\end{aligned}
$$

By the five lemma we have that $(, B)|\mathcal{L}(\Lambda) \rightarrow(, M)| \mathcal{L}(\Lambda) \rightarrow 0$ is exact. Therefore, if we show that $(, B) \mid \mathcal{L}(\Lambda)$ is finitely generated then $(, M) \mid \mathcal{L}(\Lambda)$ is also finitely generated. Since $U$ is of finite length $X=\operatorname{Hom}_{R}\left(U, I_{d}\right)$ is a $\Lambda^{\mathrm{op}}$-module such that $\operatorname{Hom}_{R}\left(X, I_{d}\right)=U$. By Proposition 3.8 we have that $\operatorname{Ext}^{1}(, U) \mid \mathcal{L}(\Lambda) \simeq$ $\operatorname{Ext}^{1}(, Y) \mid \mathcal{L}(\Lambda)$ where $Y$ is the $\Lambda$-lattice $\operatorname{Hom}_{R}\left(\Omega^{d} X, R\right)$. This isomorphism induces an isomorphism $\operatorname{Ext}^{1}(L, U) \simeq \operatorname{Ext}^{1}(L, Y)$. Hence corresponding to the element $0 \rightarrow U \rightarrow B \rightarrow L \rightarrow 0$ in $\operatorname{Ext}^{1}(L, U)$ is the element $0 \rightarrow Y \rightarrow Z \rightarrow L \rightarrow 0$ in $\operatorname{Ext}^{1}(L, Y)$. Thus we get the following exact diagram.

$$
\begin{aligned}
& 0 \rightarrow(, Y)|\mathfrak{L}(\Lambda) \rightarrow(, Z)| \mathfrak{L}(\Lambda) \rightarrow(, L)\left|\mathfrak{L}(\Lambda) \rightarrow \operatorname{Ext}^{1}(, Y)\right| \mathfrak{L}(\Lambda) \\
& 0 \rightarrow(, U)|\mathfrak{L}(\Lambda) \rightarrow(, B)| \mathfrak{L}(\Lambda) \rightarrow(, L)\left|\mathfrak{L}(\Lambda) \rightarrow \operatorname{Ext}^{1}(, U)\right| \mathfrak{L}(\Lambda)
\end{aligned}
$$

This implies that

$$
\begin{aligned}
F & =\operatorname{Ker}\left((, L)\left|\mathcal{L}(\Lambda) \rightarrow \operatorname{Ext}^{1}(, Y)\right| \mathcal{L}(\Lambda)\right) \\
& \simeq \operatorname{Ker}\left((, L)\left|\mathcal{E}(\Lambda) \rightarrow \operatorname{Ext}^{1}(, U)\right| \mathcal{L}(\Lambda)\right)
\end{aligned}
$$

which is finitely presented since $Y$ and $Z$ are lattices. But then we have the exact sequence $0 \rightarrow(, U)|\mathcal{L}(\Lambda) \rightarrow(, B)| \mathcal{L}(\Lambda) \rightarrow F \rightarrow 0$ where $F$ and $(, U) \mid \mathcal{L}(\Lambda)$ are finitely presented, which implies that $(, B) \mid \mathcal{L}(\Lambda)$ is finitely presented since finitely presented functors are closed with respect to extensions. This finishes the proof of the theorem.

\section{REFERENCES}

1. M. Auslander, Existence theorems for almost split sequences, Ring Theory. II, Proc. Second Oklahoma Conf., Marcel Dekker, New York and Basel, 1977.

2. Functors and morphisms determined by objects, Representation Theory of Algebras (Proc. Conf. Temple Univ., Philadelphia, Pennsylvania, 1976), Lecture Notes in Pure and Appl. Math., vol. 37, Marcel Dekker, New York, 1978.

3. M. Auslander and M. Bridger, Stable module theory, Mem. Amer. Math. Soc., No. 94 (1969).

4. M. Auslander and I. Reiten, Representation theory of Artin algebras. III, Comm. Algebra 3 (1975), 239-294. 
5. __ Representation theory of Artin algebras. IV, Comm. Algebra 5 (1977), 443-518.

6. M. Auslander and S. O. Smalo, Preprojective modules over Artin algebras, J. Algebra 66 (1980), $61-122$.

7. Preprojective partitions of lattices over classical orders, Lecture Notes in Math., vol. 822, Springer-Verlag, Berlin and New York, 1981, pp. 326-344.

8. H. Bass, On the ubiquity of Gorenstein rings, Math. Z. 82 (1963), 8-28.

9. E. Cartan and S. Eilenberg, Homological algebras, Princeton Univ. Press, Princeton, N. J., 1956.

10. J. Herzog and E. Kunz, Der kanonische Modul eines Cohen-Macaulay-Rings, Lecture Notes in Math., vol. 238, Springer-Verlag, New York, 1971.

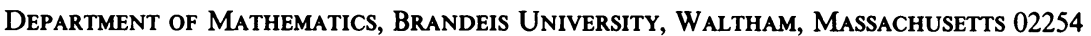

Matematisk Institutt, Universitet I TRONDheim, 7055 Dragvold, NorWay 